\title{
Intervenciones farmacéuticas: desarrollo e implementación metodo- lógica a partir de la evaluación de dos cohortes
}

\author{
Pharmaceutical interventions: methodological development and implementation from \\ the evaluation of two cohorts \\ Pamela Bertoldo ${ }^{1}$. María Gabriela Paraje ${ }^{2}$ \\ 1. Facultad de Ciencias Químicas. Universidad Católica de Córdoba. Av. Armada Argentina. Córdoba. Argentina. \\ 2. IMBIV-CONICET y Cátedra de Microbiología, Facultad de Ciencias Exactas Físicas y Naturales. Universidad Nacional de Córdoba. Av. Vélez Sarsfield \\ 299. Córdoba, Argentina.
}

\section{Artículo Original Original Article}

\section{Correspondencia} Correspondence

Pamela Bertoldo

Facultad de Ciencias Químicas. Universidad Católica de Córdoba. Av. Armada Argentina. Córdoba. Argentina pamela.bertoldo@gmail.com

Financiación

Fundings

Este trabajo fue realizado con financiamiento de la Secretaria de Investigación de la Universidad Católica de Córdoba. La Farm. Pamela Bertoldo obtuvo una beca a través del Programa Especial de Becas de Posgrado, Universidad Católica de Córdoba. La Dra. María Gabriela Paraje pertenece a la Carrera de Investigador Científico (CIC) del Consejo Nacional de Investigaciones Científicas y Técnicas (CONICET).

\section{Conflicto de interés} Competing interest

Los autores declaran que no existe conflicto de interés.

\section{Agradecimientos} Acknowledgements

Se agradece la colaboración de la Farmacéutica Virginia Trecco en la participación de las IF realizadas en la segunda cohorte.

Received: 30.12 .2014 Accepted: 10.05.2015

\section{RESUMEN}

La intervención farmacéutica (IF) son acciones que lleva a cabo el farmacéutico en la toma de decisiones en la terapia de los pacientes y en la evaluación de los resultados, con el fin de mejorar la terapia del paciente.

Objetivo. Describir y desarrollar una metodología que permita realizar y registrar intervenciones farmacéuticas (IF) en la práctica clínica.

Material y Métodos. Se realizó un estudio comparativo, transversal en dos cohortes de intervenciones farmacéuticas. Las variables de estudio se recolectan en una ficha diseñada adaptada de dos propuestas (una argentina y otra española) clasificando las IF según se realicen orientadas al medicamento, a la administración o a la prescripción médica.

Resultados. Se realizaron 460 en dos cohortes, 256 y 194 respectivamente. El 83\% de las intervenciones estuvieron centradas en el medicamento, en este grupo la IF más frecuente fue la terapia secuencial, dato que no presentó diferencias significativas entre las cohortes lo que no lleva a pensar que el instrumento y el método empleado son válidos. La aceptación de las IF fue en promedio del 95\%. Las diferencias entre el resto de los grupos fue variable según el tipo de fármacos prescriptos.

Conclusiones. En ambos períodos el instrumento de recolección permitió el registro adecuado de las IF realizadas. La IF más frecuente no presentó diferencias significativas entre ambas cohortes. En todos los casos el impacto clínico es determinante de seguridad del paciente.

Palabras clave: atención farmacéutica, impacto clínico, farmacia hospitalaria.

\begin{abstract}
Pharmaceutical interventions (PI) are actions performed in the pharmaceutical decisions in therapy of patients and the evaluation of the results, in order to improve the patient's therapy.
\end{abstract}

Aim. The aim of this study is to describe and develop a methodology to perform and record pharmaceutical interventions (PI) in clinical practice.

Material and Methods. A cross-sectional comparative study in two cohorts of pharmaceutical interventions. The study variables are collected in a form designed adapted from two proposals (one Argentina and other Spanish) PI are classified: oriented drugs, administration or medical prescription.

Results. 460 were performed in two cohorts, 256 and 194 respectively. $83 \%$ of the interventions were focused on the drug; this group was the most frequent PI sequential therapy. This information does not show significant differences between cohorts, we think that the instrument and the method are valid. Acceptance of the PI was on average 95\%. The differences between the other groups varied according to the type of prescribed drugs. 
Conclusions. In both periods the collection instrument allowed the proper registration of the PI conducted. The most common PI was no significant difference between the two cohorts. In all cases the clinical impact was decisive in patient safety.

Key words: Pharmaceutical care, clinical impact, hospital pharmacy.

\section{INTRODUCCIÓN}

En el ciclo de utilización de medicamentos, el farmacéutico debe asegurar al paciente de recibir el medicamento adecuado para su enfermedad, en la dosis correcta y en el tiempo necesario. Para esto se realiza la validación de la prescripción médica donde se efectúa una comprobación y evaluación técnica del fármaco prescripto para un paciente teniendo en cuenta el estado clínico y el medio donde está inserto $^{1}$. Este acto profesional farmacéutico se completa cuando se realiza la dispensación y el asesoramiento al paciente si fuese necesario como parte de la práctica integral que brindan los farmacéuticos en los hospitales ${ }^{2}$.

Se define como intervención farmacéutica (IF) a todas aquellas acciones que lleva a cabo el farmacéutico en forma activa, en la toma de decisiones en la terapia de los pacientes y en la evaluación de los resultados ${ }^{3}$. Estas se consideran oportunidades de mejora si logran optimizar la terapia del paciente ${ }^{4}$.

Sin embargo, y según se desprende de la revisión de varios autores $^{5-8,}$ a pesar de llevar 20 años de publicaciones de IF no existe un criterio unificado que permita clasificar en un único lenguaje las mismas. En Argentina existe una propuesta de trabajo para la clasificación de las IF propuesta por la Asociación Argentina de Farmacéuticos de Hospital, aunque todavía no hay un conceso de trabajo grupal ${ }^{9}$.

\section{Objetivo general}

El objetivo de este trabajo es describir y desarrollar una metodología para realizar IF utilizando un mismo instrumento en dos cohortes como herramienta a implementar que permita realizar y registrar IF en la práctica clínica.

\section{MATERIAL Y MÉTODOS}

Se diseñó un estudio comparativo, transversal en dos cohortes de 3 meses de duración cada una en dos años diferentes. Se consideró criterio de inclusión a todos los pacientes internados en cada cohorte de tres meses de promedio denominados « $\mathrm{A} » \mathrm{y}$ «B» en una institución con internación; con prescripciones realizadas por los médicos y validadas por el farmacéutico sobre los que se realizó una IF. Se excluyeron los pacientes pediátricos y los atendidos en salas de urgencias.
El método empleado para realizar las IF se describe a continuación: a) el farmacéutico validó la prescripción médica original (PMO), b) participa en el pase de sala donde interviene en la modificación de la prescripción original previo a la dispensación c) se acepta y modifica la PMO o no d) preparación y dispensación de la prescripción médica intervenida (Figura 1).

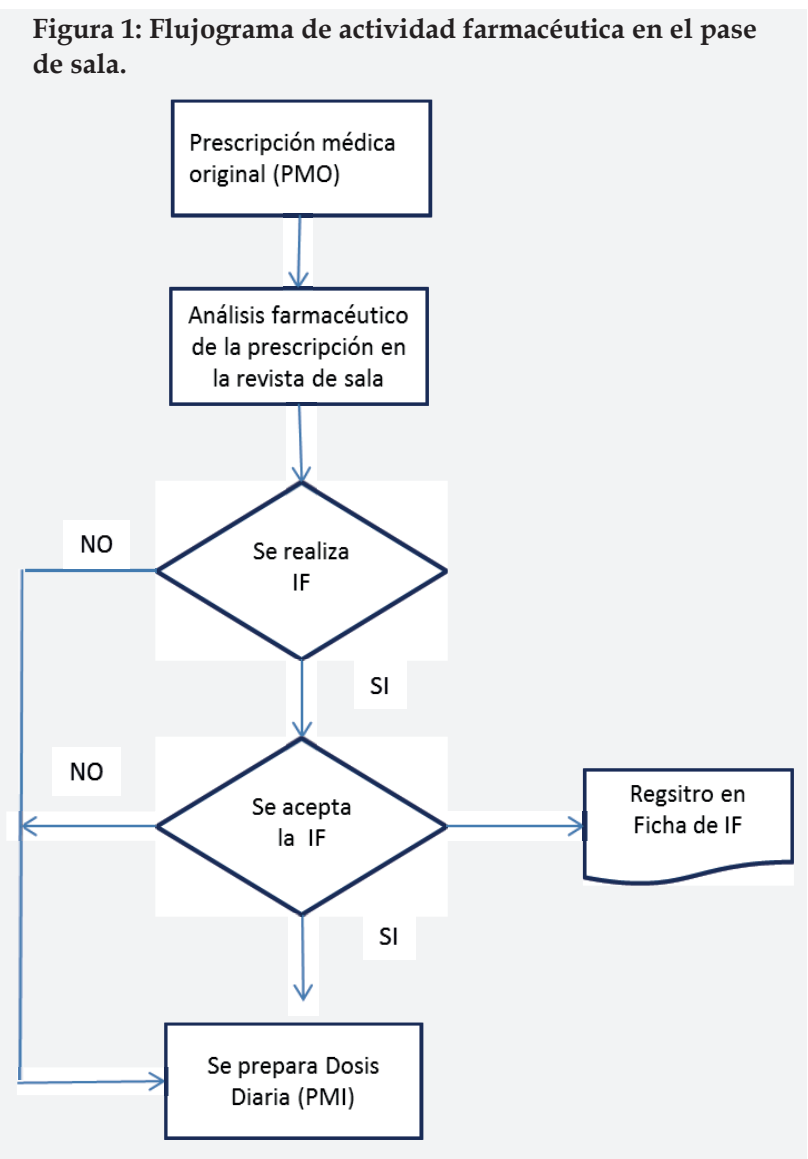

Las variables de estudio se recolectan en una ficha diseñada para este fin adaptada de la de Asociación Argentina de Farmacéuticos de Hospital $^{9}$ y basada en la propuesta de la Sociedad Española de Farmacéuticos de Hospital², las variables a considerar son: tipo de intervención, aceptación de la intervención (si / no), fármaco intervenido, costo de la prescripción original previa a la intervención y posterior a la misma. La clasificación de IF utilizada se describe a continuación adaptada a la realidad local:

\section{IF Asociadas al medicamento: el farmacéutico}

- 1a-Agrega

- 1b-Suprime

- 1c-Cambia un medicamento prescripto por el médico

- 2a-Ajusta la dosis

- 2b-Optimiza la prescripción 
- 3-Cambia la vía del medicamento prescripto(terapia secuencial)

- 4- El farmacéutico realiza una sugerencia de intercambio terapéutico cuando la PMO no considera un Medicamento de la Guía Farmacoterapéutica.

\section{IF Asociadas a la administración}

El farmacéutico corrige la forma de administración del fármaco a enfermería o realiza una acción educativa frente a la omisión de administración.

\section{IF Asociadas a la clarificación de la prescripción}

El farmacéutico interviene debido a que en la prescripción se encontró: Omisión a) de un medicamento indicado para el tratamiento de una enfermedad crónica (conciliación terapéutica) b) de información necesaria para la administración del tratamiento; o se necesitan enmiendas que aseguren la correcta dispensación y administración del fármaco prescripto $^{10}$.

Se considera en forma unitaria cada dosis intervenida y se registra al momento de la intervención el valor económico del medicamento intervenido. Las IF siempre fueron realizadas por el mismo farmacéutico. Se considera aceptada la intervención cada vez que el médico o la enfermera aceptan la sugerencia farmacéutica (se modifica la administración y/ o prescripción médica).

Se utiliza la distribución de frecuencias para la expresión de resultados de variables cualitativas y para el análisis bivariante, pruebas no paramétricas según corresponda considerando como significativa una $\mathrm{p}<0,05$.

\section{RESULTADOS}

Se realizaron un total de 450 intervenciones farmacéuticas: 256 en el periodo «A»y 194 en el «B». Se observó que la mayor proporción de intervenciones realizadas estuvieron centradas en el medicamento en ambos períodos, mostrándose un aumento de las IF asociadas a la prescripción y a la administración en el «A» con respecto al «B» Figura 2.

En la Figura 2 se muestran las IF realizadas en cada período respectivamente en función de la clasificación y descripción adaptada por los autores según monitorización terapéutica, siendo las IF orientadas al medicamento 222 y 161, grupo predominante de intervenciones realizadas en ambos períodos; 14 y 1 las orientadas a la administración y 20 y 32 las orientadas a la prescripción incluyéndose en este grupo aquellas que contemplan en forma directa la intervención asociadas a la seguridad del paciente.
En ambas fases la IF más frecuente fue la 3 considerándose semejantes en ambas situaciones $(p=0,27)$. Sin embargo se presentaron diferencias entre las IF que siguieron en orden de frecuencia en ambas fases, siendo para la fase 1 la $1 \mathrm{~B}$ y $2 \mathrm{~A}$ las que completaron los 3 primeros puestos mientras que para la fase 2 fueron la 4 y la 6B.

Figura 2: IF realizadas en cada cohorte según la clasificación asociada al medicamento, a la prescripción y a la administración del fármaco.

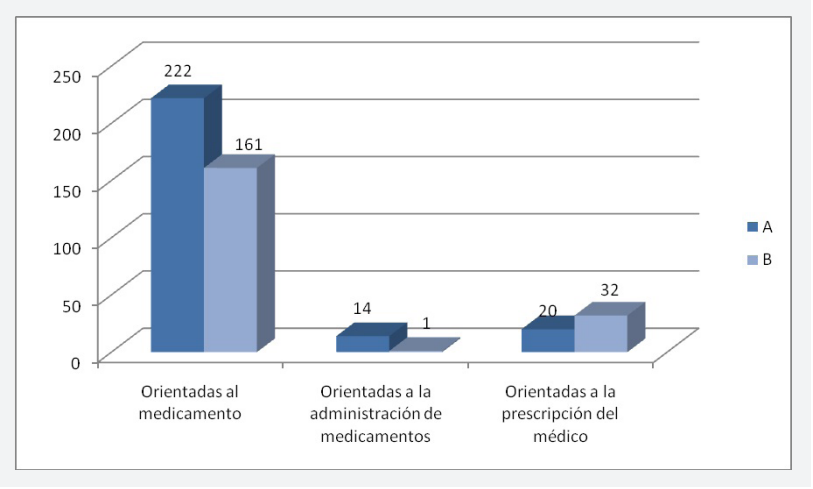

La distribución de frecuencias para todo el conjunto se describe en la Tabla 1 así como los valores de «p» para cada caso. En ambos periodos la aceptación de las IF fue el del 96 y $94 \%$ respectivamente (periodo A $247 / 256$, periodo B 183/194).

\section{DISCUSIÓN}

Como en este estudio, otros autores realizan IF a partir de la dispensación por dosis unitarias ${ }^{11,12}$; encontrándose en este trabajo concordante la cantidad de intervenciones realizadas y aceptadas. A diferencia de otros autores ${ }^{4}$, el porcentaje de IF aceptadas respecto del total de IF realizadas fueron en el día de realizada la prescripción debido a que las mismas se realizan durante el pase de sala al renovar la indicación médica.

Sin embargo, en discordancia con otros autores se incorpora la clarificación de la prescripción debido a que forma parte de un número importante (cuali y cuantitativamente) de las intervenciones realizadas en este estudio (IF 6B, 8 y 18 en cohorte «A» $\mathrm{y}$ «B» respectivamente) lo cual tiene impacto además en la seguridad del paciente.

Las IF asociadas a la administración y a la clarificación de la prescripción crecieron en la cohorte «B» demostrando que la intervención del farmacéutico es más fácil cuanto más integrado está en el equipo asistencial aunque no se encontraron diferencias significativas entre ambas. Esto demuestra en que en ninguna cohorte se hizo caso omiso a la IF planteada. 
Tabla 1: Comparación por cohorte «A» $\mathrm{y}$ «B» de los grupos monitorización-administración-prescripción y su significancia estadística.

\begin{tabular}{|c|c|c|c|c|}
\hline & Tipo de intervención & Cohorte A & Cohorte B & $\begin{array}{l}\text { Valor } \\
\text { de } p\end{array}$ \\
\hline \multirow{7}{*}{ Orientada al medicamento } & 1A: Agrega un medicamento & 6 & 9 & 0,195 \\
\hline & 1B:Suprime un medicamento & 69 & 5 & $<0,005$ \\
\hline & 1C: Cambia un fármaco & 8 & 0 & 0,02 \\
\hline & 2A: Ajusta la dosis & 26 & 1 & $<0,005$ \\
\hline & 2B: Optimiza la prescripción & 8 & 3 & 0,36 \\
\hline & 3: Cambia la vía de administración & 102 & 98 & 0,27 \\
\hline & $\begin{array}{l}\text { 4: Cambia por un medicamento del listado de } \\
\text { medicamentos }\end{array}$ & 3 & 45 & $<0,005$ \\
\hline $\begin{array}{l}\text { Orientada a la administración del } \\
\text { medicamento }\end{array}$ & 5: Se realiza una indicación a enfermería & 14 & 1 & 0,006 \\
\hline \multirow{3}{*}{$\begin{array}{l}\text { Orientada a la prescripción y el } \\
\text { médico }\end{array}$} & 6A: Conciliación de medicamentos & 10 & 4 & 0,29 \\
\hline & 6B: Enmiendas o correcciones de prescripción & 9 & 18 & 0,015 \\
\hline & $\begin{array}{l}\text { 6C: Información sobre medicamentos al equipo } \\
\text { prescriptor }\end{array}$ & 1 & 10 & 0,001 \\
\hline
\end{tabular}

Diferentes autores de lengua castellana proponen la clasificación del $3^{\text {er }}$ Consenso de Granada para identificar las IF, en nuestro equipo no se utiliza la misma porque se considera que si bien la propuesta es fácilmente utilizable en la atención de pacientes ambulatorios existen Problemas Relacionados con Medicamentos (PRM) y Resultados Negativos asociados a la Medicación (RNM) que no se adaptarían completamente al ejercicio profesional del farmacéutico de hospital ${ }^{13}$.

En este trabajo como en otros queda demostrado el impacto económico de las IF realizadas aunque no es tema desarrollado en esta presentación debido a que el análisis se centra en la validación de la metodología empleada ${ }^{14-17}$.

Existen numerosas publicaciones donde el trabajo de IF se inicia a partir de la elección de un grupo terapéutico o de enfermedades o situaciones clínicas determinadas, en este trabajo se realizaron IF en todos los pacientes con dosis diaria unitaria sin realizar preselección de los mismos según criterios de riesgo o fármacos utilizado ${ }^{18}$.

Se considera limitante de este estudio el no valorar el tipo de intervenciones realizadas según idoneidad, el impacto clínico o impacto en la seguridad del paciente y la capacidad de evitar errores de las mismas, caracterizaciones que serán tema de estudio de este grupo de trabajo en futuras presentaciones ${ }^{19}$. Si bien puede considerarse una limitación el no utilizar prescripción electrónica, es una realidad en Argentina que la misma no está difundida ni implementada en los hospitales públicos, por lo que no puede ser con- siderada como una herramienta actualmente real y superadora para realizar IF.

En este trabajo todas las comunicaciones fueron verbales a diferencia de otros autores que utilizan también la forma escrita por las características del hospital. Se considera una ventaja ya que fue esta actividad realizada durante el pase de sala lo que precisamente logra modificar una prescripción antes de realizarse efectivamente.

\section{CONCLUSIONES}

Las IF se pudieron registrar en ambas cohortes al emplear la ficha de recolección planteada. La IF más frecuente (IF3) no presentó diferencias estadísticamente significativas entre ambas cohortes lo que no lleva a pensar que el instrumento y el método empleado son válidos. Las diferencias entre el resto de los grupos fue variable lo que hace pensar que estas modificaciones están sujetas a la actividad propia del farmacéutico y la relación establecida con médico y el equipo de salud. En todos los casos el impacto clínico es determinante de seguridad del paciente.

\section{REFERENCIAS}

1. Garbayo, JLM. El farmacéutico y la «validación de la prescripción médica» Rev. O.F.I.L. 2005; 15 (3) :11-12.

2. Calvo Hernández MV, Alós Almiñana M, Giráldez Deiro J, Inaraja Bobo MT, Navarro Ruiz A, Nicolás Picó J. Bases de la atención farmacéutica en Farmacia Hospitalaria .Farm Hosp. 2006; 30 (2) : 120-123. 
3. Clopés. A. Intervenciones farmacéuticas. Disponible en: www.sefh.es/bibliotecavirtual/fhtomo1/cap1314.pdf. .

4. Gaspar $\mathrm{M}$ et al. Establecimiento de un indicador de calidad de atención farmacéutica. Farm Hosp. 2009; 33(6) : 296-304.

5. Rodríguez Torné G, García Esteban B, González Joga B, et al. Impacto clínico y económico de las intervenciones Farmacéuticas. Revista Cubana de Farmacia 2011; 45(1) : 50-59.

6. Farré Riba R, Clopés EA, Sala Esteban ML, et al. Intervenciones Farmacéuticas (parte I) Metodología y evaluación. Farm. Hosp 2000; 24 (3):136-144.

7. Martí Gil C, Sanz Ferrando M. J, Aznar Prats J. Impacto de las actuaciones farmacéuticas realizadas en un servicio de farmacia hospitalaria. Pharm Care Esp. 2011; 13(2) : 66-73.

8. Carmona García PM, García Cortez E, Lacruz Gimeno P, Evaluación de un programa de atención farmacéutica en unidades de hospitalización con dispensación individualizada de medicamentos en dosis unitaria. Farm. Hosp.2001; 25 (3) : 156-163.

9. Consultado en: Asociación Argentina de Farmacéuticos de Hospital http://www.aafhospitalaria.org.ar/pdf/intervencion_farma.pdf

10. Delgado Sánchez O, Martínez López I, Crespi Monjo M, Serra Soler G. Conciliación de la medicación: asumamos la responsabilidad compartida. Farm Hosp. 2008; 32(2) : 63-64

11. Obaldía MC, Coloma R, González L, et al. Intervención del farmacéutico en la terapéutica hospitalaria a través del sistema de distribución de medicamentos en dosis unitarias. Farm Hosp. 1995; 19: 80-85.
12. Leape LL, Cullen DJ, Clapp MD, et al. Pharmacist participation on physician rounds and adverse drugs events in the intensive unit care. JAMA. 1999; $282:$ 267-270.

13. Comité de Consenso GIAF-UGR, GIFAF-USE, GIF-UGR. Tercer Consenso de Granada sobre Problemas Relacionados con Medicamentos (PRM) y Resultados Negativos asociados a la Medicación (RNM). Ars Pharm 2007; 48(1) : 5-17.

14. Otero MJ. Mejora de la seguridad de la utilización de los medicamentos en el ámbito hospitalario. Todo Hospital. 2005; 221: 623-632.

15. Silva-Castro MM, Tuneui Valls L, Faus M.J. Revisión sistemática sobre la implantación y la evaluación del seguimiento farmacoterapéutico en pacientes hospitalizados. Farm Hosp.2010; 34(3): 106-124.

16. Wang J, Muller R, Lucarrelli CH. A Pharmacy Intervention Program: Recognizing pharmacy's contribution to improving Patient Care. Hosp Pharm.1995; 30: 129-130.

17. Suseno M, Tedeski L, Kent S, Rough S. Impact of documented pharmacist's interventions on patient care and cost. Hosp Pharm.1998; 33: 676-681.

18. Mutnick AH, Sterba KJ, Peroutka JA, Sloan NE, Beltz EA, Sorenson MK. Cost savings and avoidance from clinical interventions. Am J Health-Syst Pharm. 1997; 54: 392-396.

19. Gorgas Torner, MQ, Odena Estradé, E, Pastor Solernou, F. Atención farmacéutica en los problemas relacionados con los medicamentos en enfermos hospitalizados. Farm Hosp. 2003; 27: $280-289$ 